\title{
Incremental relaying effect on the outage probability of correlated sources Transmission
}

\author{
Zina Chkirbene ${ }^{1}$ and Noureddine Hamdi ${ }^{2}$ \\ Université de Tunis El Manar, Ecole Nationale d'Ingnieurs de Tunis \\ LR-99-ES21 Laboratoire de Systèmes de Communications, 1002, Tunis, Tunisie \\ E-mails: ${ }^{1}$ zina.chk@gmail.com, ${ }^{2}$ noureddine.hamdi@ept.rnu.tn
}

\begin{abstract}
In this paper we investigate different relaying strategies for correlated source transmission in block Rayleigh fading channels. Outage probability close form expressions are derived for both the cases where incremental decode and forward and only simple decode and forward techniques are used in the relays. We show that when using incremental technique in the principal relay, not only we gain in terms of channel resource saving but we get also a significant outage performance improvement compared to simple decode and forward system. Another finding of this paper is that if incremental relaying technique is used in the principal relay, the system reaches a diversity order of 2 with a significant power gain compared to a two links maximum ratio combining system.
\end{abstract}

Index - Decode and forward, incremental system, Outage probaility, Slepian-Wolf.

\section{INTRODUCTION}

Cooperative communication has been recently used as a powerful technique which can fundamentally change the abstraction of a wireless link and offer significant potential advantages for wireless communication networks [1] [2].

In general, sources can be correlated and in order to exploit this correlation to reduce the communication requirements, a commonly used technique is Distributed Source Coding (DSC). This technique consists on compressing separately highly correlated sources and decoding them jointly. From a theoretical point of view, DSC is based on Slepian-Wolf and Wyner-Ziv compression schemes [3], [4].

The correlation between the two information sources is assumed to be expressed by the bit flipping model, where the information bits transmitted from the second transmitter are the flipped version of the information bits transmitted from the first one, with a probability $p e$.

In [5], the authors studied the outage performance for a direct link system when two correlated sources are used based on slepian-wolf theorem. However, these studies have not been done for relaying systems. Thus, we propose in this paper a model including DSC in deocde and forward (DF) system by combining the data using both maximum ratio combining (MRC) and slepian-wolf technique. To reduce the effect of error propagation, we introduce also incremental relaying technique which shows its ability to enhance the diversity order of the system.

In this paper, the main purpose is to derive the theoretical outage probability of correlated sources transmitting with the intermediate of relays using decode and forward or incremental relaying technique. The effect of the correlation factor and of the choice of the threshold are also investigated.

The paper is organized as follows. In section II, the system model is described. In section III, we proceed to the outage probability analysis of the system. Section IV validates the analysis by simulation results. Finally, section VI summarizes and concludes the paper.

\section{SYSTEM MODEL}

The adopted system model in this paper is described in Figure1 and consists of two correlated sources ( $S 1$ and $S 2$ ) transmitting their data ( $b 1$ and $b 2$ ) to one destination $(D)$ by the intermediate of two relays $R 1$ and $R 2$.

The communication is supposed to be done over channels with a slow and frequency-flat Rayleigh fading coefficients $h_{X Y}$. Where $X$ and $Y$ denotes the different system nodes. We assume that all the additive white Gaussian noise (AWGN) terms in the 6 links $\left(S_{1} D, S_{1} R_{1}, R_{1} D, S_{2} D, S_{2} R_{2}\right.$ and $R_{2} D$ ) have equal variance $N_{0}$ and all the channels coefficients are independent. All terminals are equipped with a single antenna.

To understand the effect of path-loss on the performance of the proposed techniques, we use the model (which is commonly used in the literature e.g., [2]), where $E\left(\left|h_{X Y}(n)\right|^{2}\right)=$ $\left(\frac{d_{S_{1} D}}{d_{X Y}}\right)^{\alpha}$.

Where $d_{X Y}$ stands for the distance between the nodes $X$ and $Y, \alpha$ is the path-loss exponent and $E($.$) denotes the$ statistical average operator.

We denote by $\gamma_{X, Y}$ and $\lambda_{X, Y}$ the instantaneous received SNR from the link $\mathrm{X}-\mathrm{Y}$ and its inverse respectively.

For each source, the transmission is done in two time slots. Concerning $S 1$, in the first time slot, it sends its signal to $R 1$ and $D$. Then, in the second time slot, two different models are used. For the first one, denoted by "simple decode and forward" technique, the relay $R 1$ directly forwards its data to $D$. In the second model, denoted by "incremental" technique, the relay waits for a feedback from the destination to decide if it is needed to forward the data based on the comparison of the received SNR for the link $S_{1} D$ to a threshold $\gamma_{0}$. Then, if two signals are received, they are combined using MRC technique. The same operation is done for $S 2$ using time multiplexing (4 total time slots) or frequency multiplexing ( $S 1$ and $S 2$ send on different frequencies). 


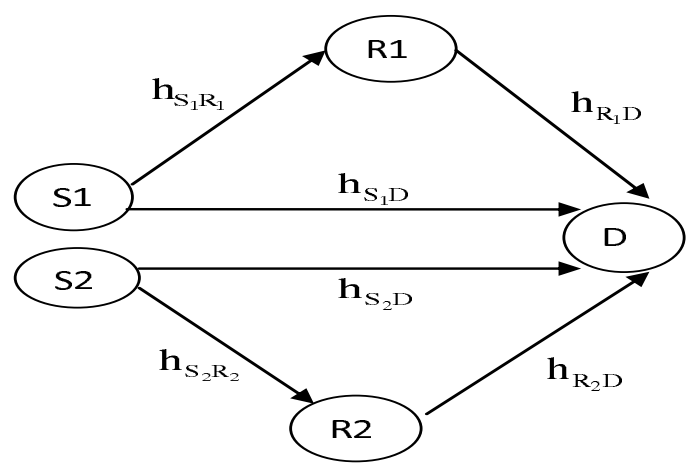

Fig. 1. System model

Then, the data received from $S 1$ and $S 2$ are combined at the destination using slepian wolf to recuperate the original data sent by $S 1$.

Since for each source, the received signals are independently combined in a first step using MRC, the system model is equivalent to the one presented by figure 2 where $\gamma_{e q_{1}}$ and $\gamma_{e q_{2}}$ denotes the equivalent received SNRs for the system transmitting $b 1$ and $b 2$ respectively.

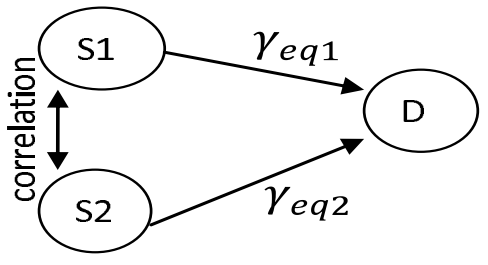

Fig. 2. Equivalent system model

\section{OUTAGE ANALYSIS}

In this section, we analyze the performance of the described systems in terms of outage probability (denoted by $P_{s w}$ ). Where an outage is defined as the event of being outside the slepian wolf admissible rate regions.

The data streams $b_{1}$ and $b_{2}$ are separately encoded and transmitted to a common receiver in different phases.

Therefore, according to the theorem of Slepian-Wolf, the admissible rate region is constituted by the parts $3,4,5$ and 6 of the Figure (3) [6]. The errorfree transmission can be guaranteed only if the rate pair falls into this area. For instance, if $\mathrm{b} 1$ is transmitted at the rate $R_{1}$ which is equal to its entropy $H\left(b_{1}\right)$, then $b_{2}$ can be transmitted at the rate $R_{2}$ which is less than its entropy $H\left(b_{2}\right)$, but must be greater than their conditional entropy $H\left(b_{2} \mid b_{1}\right)$,More specifically, R1 and R2 must meet theses equations:

$$
\begin{array}{r}
R_{b 1} \geq H\left(b_{1} \mid b_{2}\right) \\
R_{b 2} \geq H\left(b_{2} \mid b_{1}\right) \\
R_{b 1}+R_{b 2} \geq H\left(b_{1}, b_{2}\right)
\end{array}
$$

With $H(b 1, b 2)$ designating the entropy of the correlated bit streams $b 1$ and $b 2$.
We adopt in our study symmetric binary sources $(p(1)=$ $p(0)=0.5)$. We have that $H(b 1)=H(b 2)=1, H(b 1 \mid b 2)=$ $H(b 2 \mid b 1)=H(p e), H(b 1, b 2)=1+H(p e)$ with $H(p e)=$ $-\operatorname{pelog}_{2}(p e)-(1-p e) \log _{2}(1-p e)$. The SNR threshold is equal to:

$\gamma[H]=2^{R_{c} \cdot H}-1$

Where $R_{c}$ represents the rate taking into account the channel coding scheme and the modulation format.

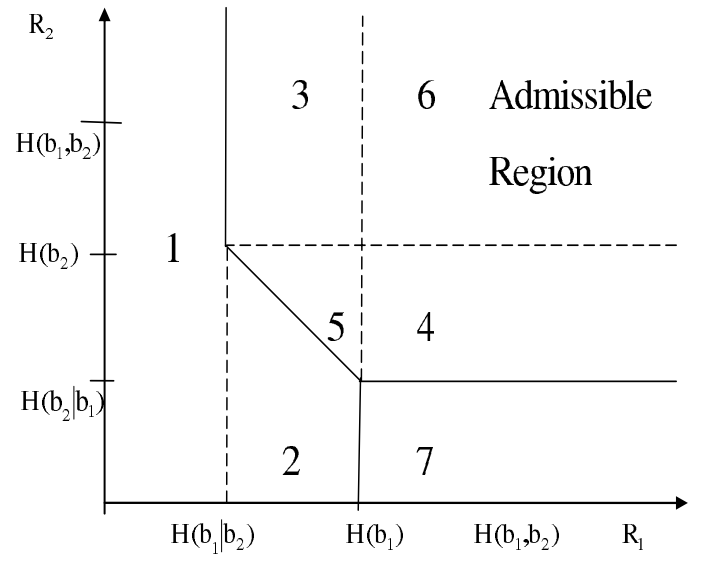

Fig. 3. Slepian-wolf admissible regions

Fig 3 shows that the possible rate regions for Slepian-Wolf when two correlated sources are considered. These regions are divided into 7 parts. It is well known that the admissible rate regions for Slepian-Wolf are the parts 3,4,5and6 [7]. However, in this article and as in [5], part 7 is also included in the admissible region, because the receiver tries only to recover $b 1$. Thus, only part 1 and 2 represent the inadmissible rate region. Therefore, the outage probability can be expressed as follows:

$$
P_{s w}=P_{1}+P_{2}
$$

where $P_{1}$ and $P_{2}$ denote respectively the probabilities that the instantaneous SNR pair $\gamma_{e q_{1}}$ and $\gamma_{e q_{2}}$ fall in the inadmissible regions 1 and 2.

Mathematically, $\mathrm{P} 1$ is defined as:

$$
P_{1}=\int_{\gamma_{e q_{1}}=\gamma[0]}^{\gamma\left[H\left(b_{1} \mid b_{2}\right)\right]} \int_{\gamma_{e q_{2}}=\gamma[0]}^{\gamma[\infty]} f_{\gamma_{e q_{1} \gamma_{e q_{2}}}}(x, y) d x d y
$$

where $f_{\gamma_{e q_{1}} \gamma_{e q_{2}}}(x, y)$ denotes the joint pdf of $\gamma_{e q_{1}}$ and $\gamma_{e q_{2}}$. With the assumption that $\gamma_{e q_{1}}$ and $\gamma_{e q_{2}}$ are statistically independent, 2 becomes:

$$
P_{1}=\int_{\gamma[0]}^{\gamma\left[H\left(b_{1} \mid b_{2}\right)\right]} \int_{\gamma[0]}^{\gamma[\infty]} f_{\gamma_{e q_{1}}}(x) f \gamma_{e q_{2}}(y) d x d y
$$

Concerning $P_{2}$, it can be defined as follows

$$
P_{2}=\int_{\gamma\left[H\left(b_{1} \mid b_{2}\right)\right]}^{\gamma\left[H\left(b_{1}\right)\right]} \int_{\gamma[0]}^{\gamma\left[H\left(b_{1}, b_{2}\right)-H\left(\gamma_{e q_{1}}\right)\right]} f_{\gamma_{e q_{1}}}(x) f \gamma_{e q_{2}}(y) d x d y
$$

In the rest of this section, we derive approximated outage expressions for the three proposed models based on the equations $(1,3$ and 4$)$. 


\section{A. Simple DF- Simple DF}

In this part, the two relays $R 1$ and $R 2$ are supposed to forward directly the received data stream independently from the direct links situations.

Concerning the data stream $b 1$, if the relay $R 1$ decoded successfully $b 1$, the destination combines the data coming on the links $S_{1} D$ and $R_{1} D$ using $M R C$. Therefore, the equivalent received SNR for the first source is $\gamma_{e q 1} \triangleq \gamma_{M R C 1}=$ $\gamma_{S_{1}, D}+\gamma_{R_{1}, D}$. Consequently, the pdf of $\gamma_{e q 1}$ becomes in this case:

$$
f_{\gamma_{M R C_{1}}}(x)=\alpha_{1}\left(e^{-\lambda_{R_{1} D}} x-e^{-\lambda_{S_{1} D}} x\right)
$$

where

$$
\alpha_{1}=\frac{\lambda_{R_{1} D} \lambda_{S_{1} D}}{\lambda_{R_{1} D}-\lambda_{S_{1} D}}
$$

However, if $R 1$ was unable to decode $b 1$, it has been proven in [8],[9] that the error probability at the destination can be approximated by 0.5 . This is equivalent to say that the channel was so bad that the transmission didn't give any information on $b 1$. Therefore, for this case, we can approximate the equivalent SNR by 0. i.e. $\gamma_{e q 1} \approx 0$.

Concerning the bit stream $b 2$. By analogy to what has been done for $b 1$ and according to the success or failure of $R 2$ to decode $b 2$, the expressions (5 and III-A) are extended to the stream $b 2$ by replacing respectively $\gamma_{M R C 1}$ and $\alpha 1$ by $\gamma_{M R C 2}=\gamma_{S 2, D}+\gamma_{R 2, D}$ and $\alpha_{2}=\frac{\lambda_{R_{2} D} \lambda_{S_{2} D}}{\lambda_{R_{2} D}-\lambda_{S_{2} D}}$.

To get the expression of $P 1$, we have to consider the following two cases:

1) $H\left(b_{1} \mid b_{2}\right)=0$ i.e. $H(p e)=0$ :

In this case, there will be no longer a region 1 and concequently $P 1=0$.

2) $H\left(b_{1} \mid b_{2}\right) \neq 0$ :

If the relay R1 decode unseccessfully the symbol, the system will be always in the zone 1,therefore

$$
\begin{aligned}
& P_{1}=\alpha_{1}\left(1-P_{S_{1} R_{1}}\right)\left(\frac{1-e^{-\lambda_{R_{1} D} \gamma[H(p e)]}}{\lambda_{R_{1} D}}-\frac{1-e^{-\lambda_{S_{1} D} \gamma[H(p e)]}}{\lambda_{S_{1} D}}\right) \\
& +P_{S_{1} R_{1}}
\end{aligned}
$$

where $P_{S_{1} R_{1}}$ denotes the probability of error in the relay $R 1$. From [10], we get the expression of $P_{S_{1} R_{1}}$ :

$$
P_{S_{1} R_{1}}=a\left(1-\sqrt{\frac{b \cdot \lambda_{S_{1} R_{1}}}{1+b \cdot \lambda_{S_{1} R_{1}}}}\right)
$$

where $(a, b)$ are constants depending on the type of modulation

Concerning $P_{2}$, we must consider first the two cases where $R 2$ decoded successfully or not $b 2$ :

1) Case $1: R 2$ decoded unseccefully $b 2$

In this case, being in zone 2 is equivalent to have $H\left(b_{1} \mid b_{2}\right) \leq R_{1} \leq H\left(b_{1}\right)$, therefore $P 2$ (denoted in this case by $P_{2_{1}}$ ) becomes:

$$
\begin{aligned}
P_{2_{1}} & =\int_{\gamma_{\left[H\left(b_{1} \mid b_{2}\right)\right]}^{\gamma_{\left[H\left(b_{1}\right)\right]}} f_{\gamma_{e q_{1}}}(x) d x} \\
& =\alpha_{1}\left(\frac{e^{-\lambda_{R_{1} D} \gamma_{[H(p e)]}}-e^{-\lambda_{R_{1} D} \gamma_{\left[H\left(b_{1}\right)\right]}}}{\lambda_{R_{1} D}}-\right. \\
& \left.\frac{e^{-\lambda_{S_{1} D} \gamma_{[H(p e)]}}-e^{-\lambda_{S_{1} D} \gamma_{\left[H\left(b_{1}\right)\right]}}}{\lambda_{S_{1} D}}\right)
\end{aligned}
$$

2) Case 2:If $R 2$ decoded successfully the symbol

$$
\begin{aligned}
P_{2_{2}} & =\alpha_{2} \int_{\gamma\left[H\left(b_{1} \mid b_{2}\right)\right]}^{\gamma\left[H\left(b_{1}\right)\right]} f_{\gamma_{e q_{1}}}(x) \\
& \left(\frac{1-e^{\left.-\lambda_{R_{2} D} \gamma_{[} H\left(b_{1}, b_{2}\right)-H(x)\right]}}{\lambda_{R_{2} D} D}\right. \\
& \left.-\frac{1-e^{\left.-\lambda_{S_{2} D}-\gamma_{[} H\left(b_{1}, b_{2}\right)-H(x)\right]}}{\lambda_{S_{2} D}}\right) d x
\end{aligned}
$$

Moreover, $f_{\gamma_{e q_{1}}}(x)$ may take different expressions depending on the success or failur of $R 1$ to deocode $b 1$ and wether $H(p e)=0$ or not:

Case $2.1 H(p e) \neq 0$ :

Case 2.1.1 R1 decoded unsuccessfully the symbol: the system will not be able to be in zone 2 since $\gamma_{e q_{2}} \approx 0 \leq \gamma[H(p e)]$ and $P_{2_{2.1 .1}}=0$

Case 2.1.2 R1 decoded successfully the symbol:

By using the expressions of $f_{\gamma_{M R C 1}}$ in (eq. 5) and its extension for the source $s 2, P_{2}$ becomes:

$$
\begin{aligned}
P_{2_{2,1,2}} & =\alpha_{1} \cdot \alpha_{2} \cdot\left[\left(\frac{e^{-x \cdot \lambda_{R_{1} D}}}{\lambda_{R_{1} D}}+\frac{e^{-x \cdot \lambda_{S_{1} D}}}{\lambda_{S_{1} D}}\right)\left(\frac{1}{\lambda_{R_{2} D}}-\frac{1}{\lambda_{S_{2} D}}\right)+\right. \\
& \frac{-e^{-\lambda_{R_{2} D}} \cdot F_{1}\left(\lambda_{R_{1} D}, \lambda_{R_{2} D} \cdot 2^{R_{c} \cdot H\left(b_{1}, b_{2}\right)}\right)}{\lambda_{R_{2} D}}+ \\
& \frac{e^{-\lambda_{R_{2} D}} \cdot F_{1}\left(\lambda_{S_{1} D}, \lambda_{R_{2} D} \cdot 2^{R_{c} \cdot H\left(b_{1}, b_{2}\right)}\right)}{\lambda_{R_{2} D}}- \\
& \frac{-e^{-\lambda_{S_{2} D} D} \cdot F_{1}\left(\lambda_{R_{1} D}, \lambda_{S_{2} D} \cdot 2^{R_{c} \cdot H\left(b_{1}, b_{2}\right)}\right)}{\lambda_{S_{2} D}}+ \\
& \left.\frac{e^{-\lambda_{S_{2} D}} \cdot F_{1}\left(\lambda_{S_{1} D}, \lambda_{S_{2} D} \cdot 2^{R_{c} \cdot H\left(b_{1}, b_{2}\right)}\right)}{\lambda_{S_{2} D}}\right]_{\gamma\left[H\left(b_{1}\right)\right]}^{\gamma\left[H\left(b_{1} \mid b_{2}\right)\right]} \\
& \triangleq\left[P_{c}\right]_{\gamma\left[H\left(b_{1}\right)\right]}^{\gamma\left[H\left(b_{1} \mid b_{2}\right)\right]} \\
& \text { where, } F_{1}(c 1, c 2)=\int e^{-c 1 \cdot x-\frac{c 2}{1+x}} d x .
\end{aligned}
$$

Case 2.2 $H(p e)=0$ :

Case 2.2.1 R1 decoded unsuccessfully the symbol Therefore, being in zone 2 is equivalent to $0 \leq$ $R_{2} \leq H\left(b_{2}\right)$. Hence, $P_{2}$ becomes:

$$
P_{2,2,1}=\alpha_{2}\left(\frac{1-e^{-\lambda_{R_{2} D \cdot \gamma\left[H\left(b_{2}\right)\right]}}}{\lambda_{R_{2} D}}-\frac{1-e^{-\lambda_{S_{2} D \cdot \gamma\left[H\left(b_{2}\right)\right]}}}{\lambda_{S_{2} D}}\right)
$$

Case 2.2.2 R1 decoded successfully the symbol, Nothing changes compared to the case 2.1.2. Therefore, $P_{2_{2,2,2}}=P_{2_{2,1,2}}$.

Finally, by combining the different expressions of $P_{2}$ for the different cases, we conclude its genral expression: 
If $H(p e)=0$ :

$P_{2}=P_{S R_{2}} P_{2_{1}}+\left(1-P_{S R_{2}}\right)\left(P_{S R_{1}} P_{2_{2,2,1}}+\left(1-P_{S R_{1}}\right) P_{2_{2,2,2}}\right)$

If $H(p e) \neq 0$ :

$P_{2}=P_{S R_{2}} P_{2_{1}}+\left(1-P_{S R_{2}}\right)\left(1-P_{S R_{1}}\right) P_{2_{2,1,2}}$

\section{B. incremental DF-simple $D F$}

To reduce the effect of error propagation caused by the use of DF technique, we assume in this section that the first relay $R_{1}$ uses incremental technique and that the second one $R_{2}$ still uses just decode and forward technique. Therefore, $f_{\gamma_{e q_{2}}}$ still have the same expression as (5).

Concerning the data stream $b 1$, we proved in the previous part that $\gamma_{e q_{1}}$ can be approximated by 0 if the relay $R_{1}$ decoded unsuccessfully the symbol,therefore,to simplify the analysis, we suppose that in such conditions, $f_{\gamma_{e q_{1}}}(x)=$ $\frac{1_{[0, \epsilon]}(x)}{\epsilon}$, where $\epsilon<<1$. Therefore, the general expression of $f_{\gamma_{e q_{1}}}^{\epsilon}(x)$ becomes:

$$
\begin{aligned}
f_{\gamma_{e q_{1}}}(x) & =P\left(\gamma_{S_{1}, D} \leq \gamma_{0}\right)\left(1-P_{S_{1}, R_{1}}\right) f_{\gamma_{M R C_{1}}}(x) \\
& +P\left(\gamma_{S_{1}, D} \leq \gamma_{0}\right) P_{S_{1}, R_{1}} f_{\gamma e q_{1}}(x) \\
& +P\left(\gamma_{S_{1}, D}>\gamma_{0}\right) f_{\gamma_{S_{1}, D}}\left(x \mid \gamma_{S_{1}, D}>0\right) \\
& =1_{\left[\gamma_{0}, \infty\right]}(x) \lambda_{S_{1}, D} e^{-\lambda_{S_{1}, D} x} \\
& +\beta_{1}\left(1_{\left[0, \gamma_{0}\right]}(x)\left(e^{-\lambda_{R_{1}, D} x}-e^{-\lambda_{S_{1}, D} x}\right)\right. \\
& \left.+1_{\left[\gamma_{0}, \infty\right]}(x) \beta_{2} e^{-\lambda_{R_{1}, D} x}\right)+\frac{1_{[0, \epsilon]}(x) \cdot \beta_{3}}{\epsilon}
\end{aligned}
$$

where

$$
\begin{aligned}
& \beta_{1}=\frac{\left(1-P_{S, R_{1}}\right) \lambda_{S_{1}, D} \lambda_{R_{1}, D}}{\lambda_{S_{1}, D}-\lambda_{R_{1}, D}} \\
& \beta_{2}=1-e^{\left(\lambda_{S_{1}, D}-\lambda_{R_{1}, D}\right) \cdot \gamma_{0}} \\
& \beta_{3}=P_{S_{1}, R}\left(1-e^{\lambda_{S_{1}, D} \cdot \gamma_{0}}\right)
\end{aligned}
$$

To get the expression of $P_{1}$, two cases must be considered: Case 1 If $\gamma_{0} \leq \gamma\left[H\left(b_{1} \mid b_{2}\right)\right]$

$$
\begin{gathered}
P_{1}=\left(e^{-\lambda_{S_{1}, D} \gamma_{0}}-e^{-\lambda_{S_{1}, D} \gamma\left[H\left(b_{1} \mid b_{2}\right)\right]}\right)+ \\
\beta_{1}\left(\frac{\left(1-e^{-\lambda_{R_{1}, D} \gamma_{0}}\right)}{\lambda_{R_{1}, D}}-\frac{\left(1-e^{-\lambda_{S_{1}, D} \gamma_{0}}\right)}{\lambda_{S_{1}, D}}\right)+ \\
\beta_{2}\left(\frac{e^{-\lambda_{R_{1}, D} \gamma_{0}}-e^{-\lambda_{R_{1}, D} \gamma\left[H\left(b_{1} \mid b_{2}\right)\right]}}{\lambda_{R_{1}, D}}\right)+\beta_{3}
\end{gathered}
$$

Case 2 If $\gamma_{0}>\gamma\left[H\left(b_{1} \mid b_{2}\right)\right]$

$$
\begin{aligned}
P_{1} & =\beta_{1}\left(\frac{\left(1-e^{-\lambda_{R_{1}, D} \gamma\left[H\left(b_{1} \mid b_{2}\right)\right]}\right)}{\lambda_{R_{1}, D}}-\frac{\left(1-e^{-\lambda_{S_{1}, D} \gamma\left[H\left(b_{1} \mid b_{2}\right)\right]}\right)}{\lambda_{S_{1}, D}}\right) \\
& +\beta_{3}
\end{aligned}
$$

Concerning $P 2$, two major cases may be considered:

Case 1 If $\gamma\left[H\left(b_{1}, b_{2}\right)-H(x)\right]=0$

In this case $x=\gamma\left[H\left(b_{1}, b_{2}\right)\right]$, it is an impossible case since $\gamma\left[H\left(b_{1}, b_{2}\right)\right]>\gamma\left[H\left(b_{1}\right)\right]=1$

Case 2 If $\gamma\left[H\left(b_{1}, b_{2}\right)-H(x)\right] \neq 0$

Case 2.1 If $H\left(b_{1} \mid b_{2}\right) \neq 0$ (To simplify the analysis, only this case is considered and the case $2.2 H\left(b_{1} \mid b_{2}\right)=0$ is not taken into account)
Case 2.1.1 If $\gamma_{0} \leq \gamma\left[H\left(b_{1} \mid b_{2}\right)\right]$

$$
\begin{aligned}
P_{2} & =\left[( 1 - P _ { S _ { 2 } } ) \alpha _ { 2 } \left(\left(-e^{-\lambda_{S_{1} D} x}-e^{-\lambda_{R_{1} D} x} \frac{\beta_{1} \cdot \beta_{2}}{\lambda_{S_{1} D}}\right)\right.\right. \\
& \left(\frac{1}{\lambda_{R_{2} D}}-\frac{1}{\lambda_{S_{2} D}}\right)-\frac{\lambda_{S_{1} D}}{\lambda_{R_{2} D}} e^{-\lambda_{R_{2} D}} F_{1}\left(\lambda_{S_{1} D}, \lambda_{R_{2} D} 2^{R_{c}} H\left[b_{1}, b_{2}\right]\right) \\
& +\frac{\beta_{1} \beta_{2}}{\lambda_{R_{2} D}} e^{-\lambda_{R_{2} D}} F_{1}\left(\lambda_{R_{1} D}, \lambda_{R_{2} D} 2^{R_{c}} H\left[b_{1}, b_{2}\right]\right) \\
& +\left(\frac{\lambda_{S_{1} D}}{\lambda_{S_{2} D}} e^{-\lambda_{S_{2} D}} F_{1}\left(\lambda_{S_{1} D}, \lambda_{S_{2} D} 2^{R_{c}} H\left[b_{1}, b_{2}\right]\right)\right. \\
& \left.+\frac{\beta_{1} \beta_{2}}{\lambda_{S_{2} D}} e^{-\lambda_{S_{2} D}} F_{1}\left(\lambda_{R_{1} D}, \lambda_{S_{2} D} 2^{R_{c}} H\left[b_{1}, b_{2}\right]\right)\right) \\
& \left.\left.+P_{S_{2}, R}\left(e^{-\lambda_{S_{1} D} x}-\frac{\beta_{1} \beta_{2}}{\lambda_{R_{1} D}} e^{-\lambda_{R_{1} D} x}\right)\right)\right]_{\gamma\left[H\left(b_{1}\right)\right]}^{\left.\gamma\left[b_{2}\right)\right]} \\
& \triangleq\left[P_{a}\right]_{\gamma\left[H\left(b_{1} \mid b_{2}\right)\right]}^{\gamma\left[H\left(b_{1}\right)\right]}
\end{aligned}
$$

Case 2.1.2 If $\gamma_{0}>\gamma\left[H\left(b_{1}\right)\right]$

$$
\begin{aligned}
P_{2} & =\left[\left(( 1 - P _ { S , R _ { 2 } } ) \alpha _ { 2 } \beta _ { 1 } \left(\left(-\frac{1}{\lambda_{R_{1}, D}} e^{-\lambda_{R_{1}, D} x}\right.\right.\right.\right. \\
& \left.+\frac{1}{\lambda_{S_{1}, D}} e^{-\lambda_{S_{1}, D} x}\right)\left(\frac{1}{\lambda_{R_{2}, D}}-\frac{1}{\lambda_{S_{2}, D}}\right) \\
& -\frac{e^{\lambda_{R_{2}, D}}}{\lambda_{R_{2}, D}} F_{1}\left(\lambda_{R_{1}, D}, \lambda_{R_{2}, D} 2^{R_{c}} H\left[b_{1}, b_{2}\right]\right) \\
& \left.-\frac{e^{\lambda_{R_{2}, D}}}{\lambda_{R_{2}, D}} F_{1}\left(\lambda_{S_{1}, D}, \lambda_{R_{2}, D} 2^{R_{c}} H\left[b_{1}, b_{2}\right]\right)\right) \\
& +\left(\frac{e^{\lambda_{S_{2}, D}}}{\lambda_{S_{2}, D}} F_{1}\left(\lambda_{R_{1}, D}, \lambda_{S_{2}, D} 2^{R_{c}} H\left[b_{1}, b_{2}\right]\right)\right. \\
& \left.-\frac{e^{\lambda_{S_{2}, D}}}{\lambda_{S_{2}, D}} F_{1}\left(\lambda_{S_{1}, D}, \lambda_{S_{2}, D} 2^{R_{c}} H\left[b_{1}, b_{2}\right]\right)\right) \\
& +\beta_{1} \cdot P_{S_{2}, R}\left(\frac{1}{\lambda_{S_{1}, D}} e^{-\lambda_{S_{1}, D} x}\right) \\
& \left.\left.-\left(\frac{1}{\lambda_{R_{1}, D}} e^{-\lambda_{R_{1}, D} x}\right)\right)\right]_{\gamma\left[H\left(b_{1} \mid b_{2}\right)\right]}^{\gamma\left[H\left(b_{1}\right)\right]} \\
& \left.\triangleq\left[P_{b}\right]_{\gamma\left[H\left(b_{1} \mid b_{2}\right)\right]}^{\gamma[H}\right)
\end{aligned}
$$

Case 2.1.3 If $\gamma\left[H\left(b_{1}\right)\right] \geq \gamma_{0}>\gamma\left[H\left(b_{1} \mid b_{2}\right)\right]$

$$
P_{2}=\left[P_{a}\right]_{\gamma_{0}}^{\gamma\left[H\left(b_{1}\right)\right]}+\left[P_{b}\right]_{\gamma\left[H\left(b_{1} \mid b_{2}\right)\right]}^{\gamma_{0}}
$$

\section{Incremental DF- Incremental DF}

In this section, the transmission from $R 1$ and $R 2$ to $D$ are supposed to be conditioned on the feedback of the destination. Since the two relays are using incremental technique, $f_{e q_{1}}(x)$ keeps the same expression as in(11)and $f_{e q_{2}}(x)$ is obtained by replacing respectively $\beta_{1}, \beta_{2}$ and $\beta_{3}$ by $\beta_{1}^{\prime}, \beta_{2}^{\prime}$ and $\beta_{3}^{\prime}$ defined by:

$$
\begin{aligned}
\beta_{1}^{\prime} & =\frac{\left(1-P_{S_{2}, R_{2}}\right) \lambda_{S_{2}, D} \lambda_{R_{2}, D}}{\lambda_{S_{2}, D}-\lambda_{R_{2}, D}} \\
\beta_{2}^{\prime} & =1-e^{\left(\lambda_{S_{2}, D}-\lambda_{R_{2}, D}\right) \cdot \gamma_{0}} \\
\beta_{3}^{\prime} & =P_{S_{2}, R}\left(1-e^{\lambda_{S_{2}, D} \cdot \gamma_{0}}\right)
\end{aligned}
$$

The expressions of $f_{\gamma_{e q_{1}}}(x)$ and $f_{\gamma_{e q_{2}}}(x)$ depends on $\gamma_{0}$ and will include a lot of cases, especially for the calculations of $P_{2}$. 
Therefore, we suppose that $\gamma_{0} \geq \gamma\left[H\left(b_{1}\right)\right]=\gamma\left[H\left(b_{2}\right)\right]=1$. This supposition is logic because too low values of $\gamma_{0}$ refers to a system with "simple relaying-simple relaying" which is a system that we already studied in the first part. $P_{1}$ has the same expression present in (3), and it is equals to:

$$
\begin{aligned}
P_{1} & =\beta_{1}\left(\frac{1-e^{-\lambda_{R_{1}, D} \gamma\left[H\left(b_{1} \mid b_{2}\right)\right]}}{\lambda_{R_{1}, D}}-\frac{1-e^{-\lambda_{S_{1}, D} \gamma\left[H\left(b_{1} \mid b_{2}\right)\right]}}{\lambda_{S_{1}, D}}\right) \\
& +\beta_{3}
\end{aligned}
$$

Since we supposed that $\gamma_{0} \geq \gamma\left[H\left(b_{1}\right)\right]=\gamma\left[H\left(b_{2}\right)\right]=1$, we have that $\gamma[H(b 1, b 2)-H(x)] \leq \gamma_{0}$. Therefore

$$
\begin{aligned}
& P_{2}=\int_{\gamma\left[H\left(b_{1} \mid b_{2}\right)\right]}^{\gamma\left[H\left(b_{1}\right)\right]}\left(\beta _ { 1 } \beta _ { 1 } ^ { \prime } \left(\frac{1-e^{-\lambda_{R_{2}, D} \gamma\left[H\left(b_{1} \mid b_{2}\right)-H(x)\right]}}{\lambda_{R_{2}, D}}\right.\right. \\
&\left.-\frac{1-e^{-\lambda_{S_{2}, D} \gamma\left[H\left(b_{1} \mid b_{2}\right)-H(x)\right]}}{\lambda_{S_{2}, D}}\right)\left(\frac{1-e^{-\lambda_{R_{1}, D} \gamma\left[H\left(b_{1} \mid b_{2}\right)-H(x)\right]}}{\lambda_{R_{1}, D}}\right. \\
&\left.-\frac{1-e^{-\lambda_{S_{1}, D} \gamma\left[H\left(b_{1} \mid b_{2}\right)-H(x)\right]}}{\lambda_{S_{1}, D}}\right)+\beta_{1} \beta_{3}^{\prime} \\
&\left(\frac{1-e^{-\lambda_{R_{1}, D} \gamma\left[H\left(b_{1} \mid b_{2}\right)-H(x)\right]}}{\lambda_{R_{1}, D}}-\frac{1-e^{-\lambda_{S_{1}, D} \gamma\left[H\left(b_{1} \mid b_{2}\right)-H(x)\right]}}{\lambda_{S_{1}, D}}\right) d x
\end{aligned}
$$

For the simulation, this expression is evaluated using the trapezoidal numerical integration method .

\section{Simulation Results}

This section presents the simulation results which validate the analysis for the different studied scenarios where the relays use incremental relaying technique or not. Asymmetric network geometry is examined where the relay is located across the straight line connecting the source and the destination.

Fig.4 presents The slepian-wolf outage probability of a "simple DF -simple DF" system for different values of $p e$. We can remark that for low SNR, this system outperforms MRC technique with two branches. It is seen also that the smaller pe is, the better results we get in terms of outage probability. In fact, having small values of $p e$ means that the two sources are sending approximately the same data which give more chance for the destination to decode $b 1$. However, for high SNR, this system no longer outperforms MRC and gives the same outage for the different values of $p e$. In fact, this is a result of the error propagation incremental transmission technique in the relay $R 1$ based on the threshold $\gamma_{0}$.

Figure 5 shows the effect of the forwarding threshold $\gamma_{0}$ on the outage probability of the system. Very high $\gamma_{0}$ means that the destination never asks from the relay $R 1$ to forward its data which will be equivalent to have a direct link transmission for $S 1$. However for very low $\gamma_{0}, R 1$ will always forward its data. Therefore, the system gives better outage performances for low $\gamma_{0}$ compared to high $\gamma_{0}$ which can Remarque by Figure 5. However, for a particular value of $\gamma_{0}$, the system gives lower outage probability than a system where the relay always forward his data. Therefore, we conclude that the choice of $\gamma_{0}$ can control when $R 1$ forwards useful information or just

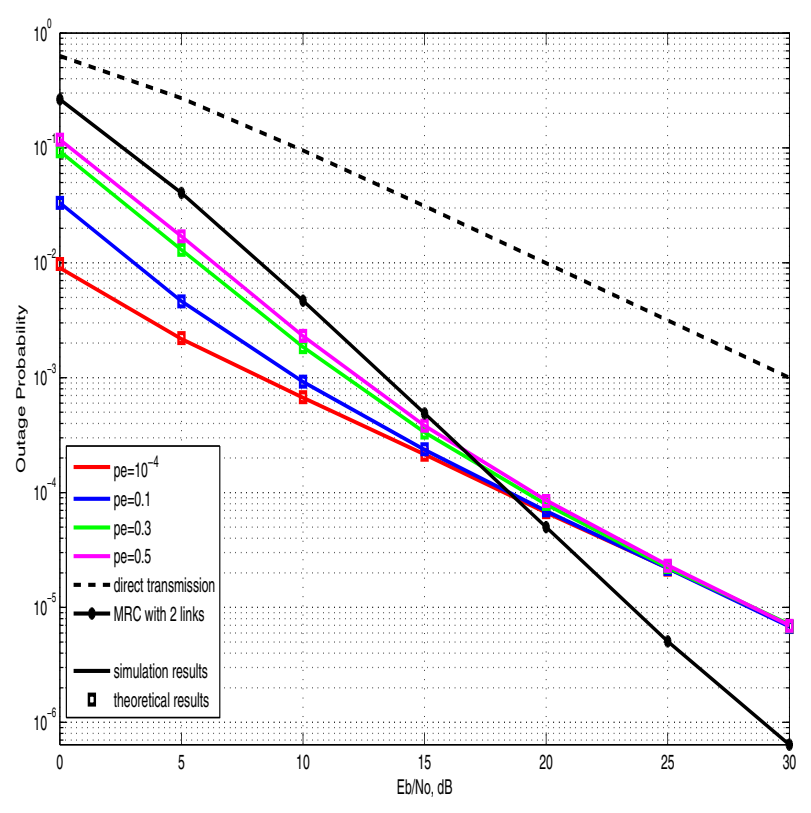

Fig. 4. The outage probability of a "simple relaying -simple relaying" system for different values of $p e$

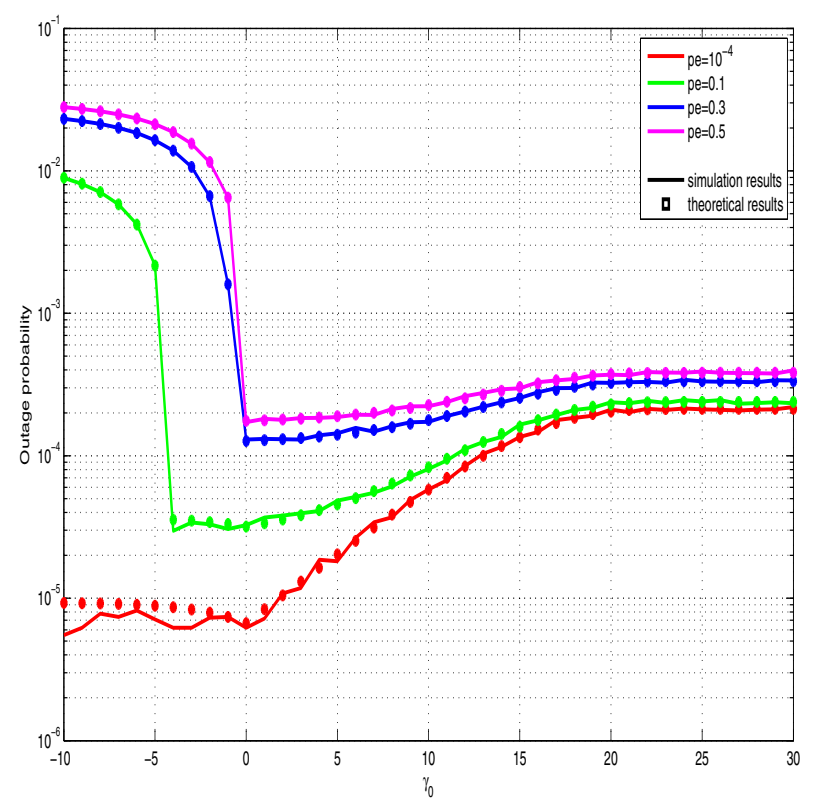

Fig. 5. effect of $\gamma_{0}$ on the outage probability of an "incremental relaying -simple relaying" system for different values ofpe and for $E b / N 0=15 d B$

cause error propagation. This best value of $\gamma_{0}$ is variable and depends on pe. However, particularly when $\frac{E b}{N 0}=15 d B$, the variation of the optimal threshold isn't important (range from $0 d B$ to $2 d B$ ). Therefore, in Figure 6, we fixed $\gamma_{0}$ to be $2 d B$ and plotted the outage probability in function of the received SNR for different values of $p e$. 


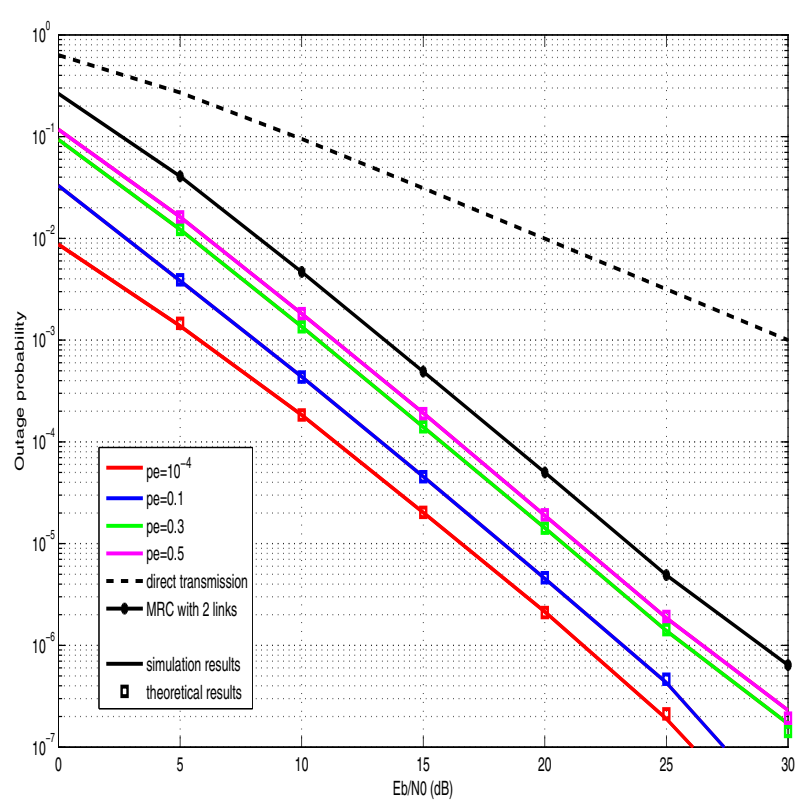

Fig. 6. The outage probability of a "incremental relaying -simple relaying" system for $\gamma_{0}=2 d B$ for different values of pe

Compared to Figure 4, we can see in 6 that adding the incremental transmission technique to $R 1$, allowed the system to outperform MRC system not only for low SNR (as for simple DF- simple DF) but also for high SNR. Figure 6 shows also that the new system has the same diversity order as an MRC system (2) which wasn't the case when $R 1$ always forwards its data.

In figure 7 , we added the incremental technique also to the relay $R 2$, no significant changes can be seen compared to a system when only $R 1$ uses the incremental technique. Therefore we conclude that incremental technique is only benefic if used in the relay $R 1$ since it is carrying the data that we aim to recover $b 1$.

\section{CONCLUSIONS}

In this work, the outage probability of a correlated source transmission system based on the Slepian-Wolf theorem has been analyzed for simple DF-simple DF, incremental DFsimple DF and incremental DF-incremental DF models. The bit-flipping model was used to express the correlation model between the two sources, and the close form expressions of the outage probability were theoretically calculated assuming that the receiver aims to decode $b 1$. We showed that simple DFsimple DF system, outperforms MRC with 2 links only for low SNR. However, when incremental relaying incremental relaying technique is used in the principal relay, beside allowing channel resource saving the outage improvement remains also for high SNR with a significant power gain compared to MRC. We showed also that the choice of the threshold is very important for the incremental transmission performance.

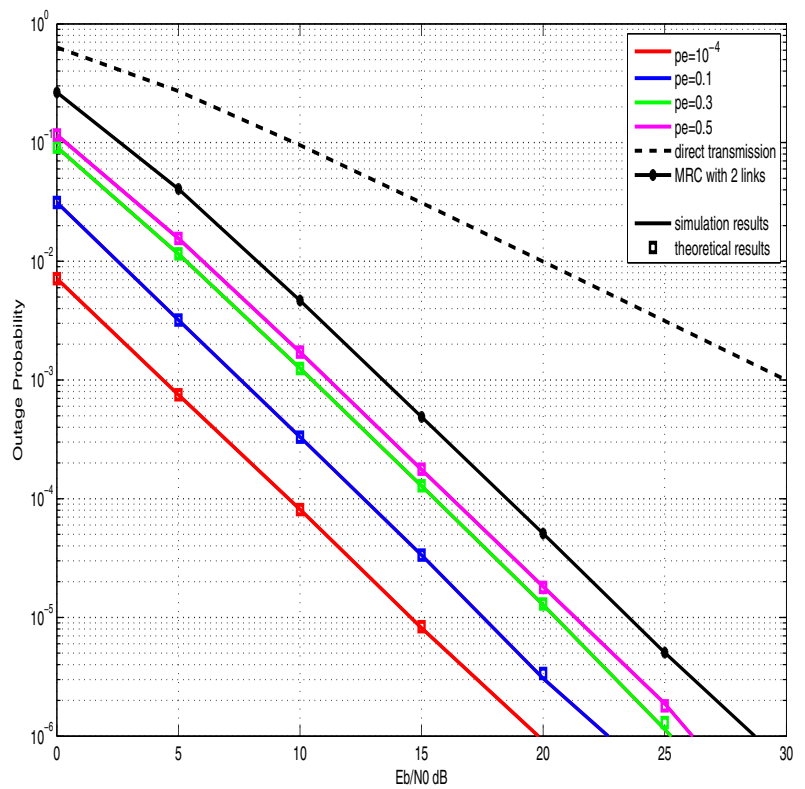

Fig. 7. The outage probability of an "incremental DF -incremental DF" system for $\gamma_{0}=2 d B$ for different values of $p e$

\section{REFERENCES}

[1] A. Nosratinia, T.E. Hunter, and A. Hedayat, "Cooperative communication in wireless networks," Communications Magazine, IEEE, vol. 42, no. 10 , pp. $74-80$, oct. 2004 .

[2] J Nicholas Laneman, David N C Tse, and Gregory W Wornell, "Cooperative Diversity in Wireless Networks : Efficient Protocols and Outage Behavior," vol. 50, no. 12, pp. 3062-3080, 2004.

[3] D. Slepian and J. Wolf, "Noiseless coding of correlated information sources," IEEE Transactions on Information Theory, vol. 19, no. 4, pp. $471-480$, jul 1973.

[4] A. Wyner and J. Ziv, "The rate-distortion function for source coding with side information at the decoder," IEEE Transactions on Information Theory, vol. 22, no. 1, pp. 1 - 10, jan 1976.

[5] Tad Matsumotol Meng Cheng, Khoirul Anwar, "Outage Analysis of Correlated Source Transmission in Block Rayleigh Fading Channels," IEEE Transactions on Communications, vol. 53, no. 5, pp. 978-984, 2012.

[6] D, J Slepian, and Wolf, "Noiseless coding of correlated information sources," IEEE Transactions on Information Theory, vol. 19, no. 4, pp. 471-480, July 1973.

[7] J, Y Garcia-Frias, and Zhaol, "Near-shannon/slepian-wolf performance for unknown correlated sources over awgn channels," IEEE Transactions on Communications, vol. 53, no. 4, pp. 555-559, 2005.

[8] A, H Adinoyi, and Yanikomeroglu, "Cooperative relaying in multiantenna fixed relay networks," IEEE Transactions on Wireless Communications, vol. 6, no. 9, pp. 533-544, Feb. 2007.

[9] P, E Herhold, G Zimmermann, and Fettweis, "A Simple Cooperative Extension to Wireless Relaying," in Int. Zurich Seminar on Communications, vol. 5, Feb. 2004.

[10] J. G. Proakis, Digital Communications, McGraw Hill, 1995. 